\title{
Feeling in control of your footsteps: Conscious gait monitoring and the auditory consequences of footsteps
}

\author{
Fritz Menzer ${ }^{1}$, Anna Brooks ${ }^{1,2}$, Pär Halje ${ }^{1}$, Christof Faller ${ }^{1}$, \\ Martin Vetterli ${ }^{1}$, and Olaf Blanke ${ }^{1}$ \\ ${ }^{1}$ Ecole Polytechnique Fédérale de Lausanne, Lausanne, Switzerland \\ ${ }^{2}$ Southern Cross University, Coffs Harbour, Australia
}

\begin{abstract}
A fundamental aspect of the "I" of conscious experience is that the self is experienced as a single coherent representation of the entire, spatially situated body. The purpose of the present study was to investigate agency for the entire body. We provided participants with performance-related auditory cues and induced online sensorimotor conflicts in free walking conditions investigating the limits of human consciousness in moving agents. We show that the control of full-body locomotion and the building of a conscious experience of it are at least partially distinct brain processes. The comparable effects on agency using audio-motor and visuo-motor cues as found in the present and previous agency work may reflect common supramodal mechanisms in conscious action monitoring. Our data may help to refine the scientific criteria of selfhood and are of relevance for the investigation of neurological and psychiatric patients with disturbance of selfhood.
\end{abstract}

Keywords: Motor awareness; Self; Agency; Sensorimotor; Auditory; Gait; Consciousness.

\section{INTRODUCTION}

In a scene from The Man Who Knew Too Much (Alfred Hitchcock, 1956), Dr Ben McKenna played by Jimmy Stewart walks down an empty street in London. He is searching for his son Hank who he believes was kidnapped by a couple in Morocco two days earlier. Dr McKenna is alone and suspects that people may be following him. Actor and audience hear footsteps that do not seem to come from Dr McKenna's feet. However, the heard footsteps slow down, stop, accelerate as Dr McKenna slows down, stops, and accelerates. Where are the footsteps coming from? Is someone following Dr McKenna? Do they reflect a loud and distorted echo of his footsteps? Dr McKenna turns around several times during that sequence but sees nobody else. Determining whether we are causing the perceptual events that we perceive or whether somebody or something else causes these events is an important function and is generally referred to as agency. How do I know whether I am causing the rhythmic sounds of footsteps? When do I begin to suspect that another agent is causing the sounds I hear?

"Actions are critical steps in the interaction between the self and the external milleu" (Jeannerod, 2007) and may reveal—especially when self-generated and not responses to external events-the intentions, states, and goals of the acting self. Moreover, such selfgenerated actions also alter the perceptual environment.

Correspondence should be addressed to: Olaf Blanke, Laboratory of Cognitive Neuroscience, Brain-Mind Institute, Station 19, Ecole Polytechnique Fédérale de Lausanne (EPFL), 1015 Lausanne, Switzerland. E-mail: olaf.blanke@epfl.ch 
Previous work has shown that agency judgments can be influenced by the manipulation of perceptual and sensorimotor cues during different phases of action execution (Farrer et al., 2008; Fourneret \& Jeannerod, 1998; Frith, Blakemore, \& Wolpert, 2000; Knoblich \& Kircher, 2004; Sato \& Yasuda, 2005). Thus, it has been tested whether discrepancies between sensory predictions and the actual sensory input (re-afference) may lead participants to judge self-generated actions as externally generated. Following early work by Nielsen (1963), this latter form of agency (or conscious action monitoring) has recently been the topic of intensive research (Fourneret \& Jeannerod, 1998; Franck et al., 2001). In these studies participants' agency was measured in response to varied sensorimotor incongruencies between visual and motor (and proprioceptive) signals (that are generally congruent during action execution). In these studies the authors investigated sensorimotor conflicts by manipulating the visual position of the participant's hand as seen on a computer screen and the participant's actual hand while a simple goal-directed motor task was carried out. Participants were asked to direct their hand to a specified target position. Direct vision of the participant's hand during the target-directed action was occluded and visual feedback of the arm movements (again as seen on a computer screen) was systematically deviated from its actual movement path. These studies showed that participants automatically aligned their hand trajectories with a visual target on the computer screen while compensating for a displayed spatial deviation. The participants were often unaware of their online movement corrections and judged many of these movements as non-deviated; these data also revealed that agency decreased with increasing sensorimotor incongruency between these visual and motor cues. In a similar experimental set-up, Franck et al. (2001) investigated the influence of temporal cues on agency judgments. The authors introduced different temporal delays between the visual position of the participant's hand (as seen on a computer screen) and the participant's actual hand while the same motor task was carried out. As observed for spatial deviations, increasing delay durations between visual and sensorimotor cues were found to decrease agency (see also Shimada, Qi, \& Hiraki, 2010).

Most previous work has focused on the investigation of performance-related visual cues on agency (Daprati et al., 1997; Farrer et al., 2003, 2008; Fourneret \& Jeannerod, 1998; Franck et al., 2001; Knoblich \& Kircher, 2004; Shimada et al., 2010; Tsakiris, Haggard, Franck, Mainy, \& Sirigu, 2005; van den Bos \& Jeannerod, 2002). Auditory action consequences may differ in their effects from visual action consequences, as the auditory detection of temporal discrepancies may be an especially powerful agency cue due to the excellent timing of auditory perception. In spite of this such auditory effects on agency have only rarely been tested (Asai \& Tanno, 2008; Knoblich \& Repp, 2009; Repp \& Knoblich, 2007; Sato \& Yasuda, 2005).

The above example from the The Man Who Knew Too Much points to a fundamental difference between the conscious monitoring of actions as tested in most of the previous agency work and conscious monitoring related to questions such as "How do I know whether the footsteps I hear are mine?" Almost all previous work on agency has focused on the investigation of performance-related sensory cues for upper limb actions (actions of fingers, hands, or arms). Yet a fundamental aspect of the "I" of conscious experience is that the self is experienced as a single coherent representation of the entire, spatially situated body, not as several separate body parts (Blanke \& Metzinger, 2009). Recently, several experimental procedures have been reported that allow one to test full-body ownership or the conscious experience of identifying with one's body and of being localized within one's body (Ehrsson, 2007; Lenggenhager, Mouthon, \& Blanke, 2009; Lenggenhager, Tadi, Metzinger, \& Blanke, 2007; Petkova \& Ehrsson, 2008) and such changes in full-body ownership have also been shown to modify the perception of tactile stimuli (Aspell, Lenggenhager, \& Blanke, 2009). Concerning agency and because the participants' body position was kept constant (except for actions of finger, hand, or arm), previous agency studies did not investigate this fundamental aspect of the global bodily self, because this requires movement of the entire body of the participant as during locomotion. Footstep-related signals during locomotion are probably one of the most common performancerelated auditory cues (alongside speech or eating) and of significant relevance for the self. This is suggested by the scene from The Man Who Knew Too Much and by clinical data in neurological patients (i.e. Blanke, Ortigue, Coeytaux, Martory, \& Landis, 2003). Moreover, walking differs from upper limb actions in several physiological ways: Gait is cyclic, more rarely immediately goal-directed, and is generally considered a highly automatic and unconscious action with important control centres in spinal cord and brainstem (Armstrong, 1988; Grillner \& Wallen, 1985). Collectively, these data suggest that agency for the full body may differ from agency for the upper limb. 
To investigate conscious action monitoring for the entire body we asked participants to make agency judgments during locomotion. For this we developed a portable device that allows the introduction of different systematic temporal delays between the participants' footsteps and the auditory consequences of those footsteps. This was combined with an analysis of the walking speed during the different tested delays. We predicted that an increase in delay should lead to a decrease in agency judgments as observed in previous arm agency studies, but that longer delays (those approaching the next footstep) would lead to increases in agency judgments.

\section{METHODS}

\section{Participants}

Eleven healthy participants with normal or corrected-to-normal hearing (five female; one lefthanded), aged 21-30 years, volunteered for the experiment. Experiments were conducted in accordance with the Declaration of Helsinki and accepted by the local ethics commission (University Hospital of Lausanne).

\section{Procedure}

After a participant's shoes were removed and replaced with experimental shoes (see below), we taped the microphone chords to the participant's trousers and the microphones to their shoes, and the backpack in which the laptop computer running the experiment software was carried and the eyewear were donned (Figure 1). In order to minimize visual contributions to the task, participants were instructed to keep their heads up and not to inspect the patterns of movement of their limbs (controlled by experimenter). Participants also wore occluding eyewear such that visual information from below the head was impaired (field of view was limited to $\sim 15^{\circ} \times \sim 15^{\circ}$ ).

After this, participants performed as many baseline trials as necessary to familiarize themselves with the experimental setup, walking, and task. They were instructed to walk continuously in a clockwise circuit prescribed by a cordoned-off hallway measuring $20 \times 4 \mathrm{~m}$ (Figure 1). Individual trials were initiated by themselves via a button on a handheld wireless device. Two other buttons were assigned as "response" buttons. Two alternative forced-choice judgments were registered before further trials could commence. Partici-
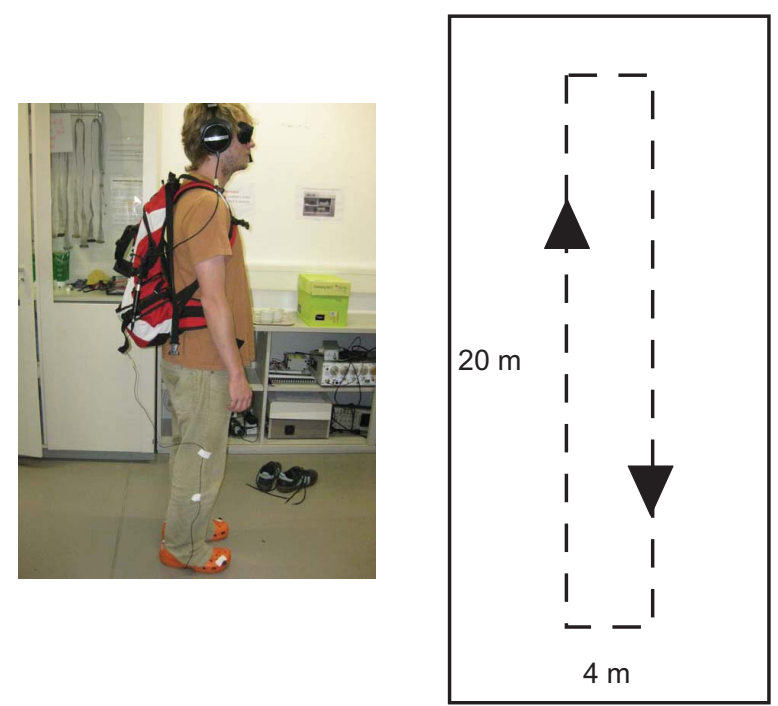

Figure 1. Experimental setup. (Left) A participant wearing the recording and stimulation system. (Right) Plan of the hallway (measuring $20 \mathrm{~m} \times 4 \mathrm{~m}$ ) where particpants were instructed to walk continuously in a clockwise circuit.

pants were instructed not to initiate trials on their approach to the turn at the end of the hallway, but rather to wait to initiate the next trial until the commencement of their walk down the length of the space (to minimize potential left-right differences in the auditory cues that would be associated with turning in a consistent (clockwise) direction). Participants were instructed to walk at a normal and relaxed speed at all times (as if they were "taking a stroll along a footpath") and informed that trials would be presented in four blocks, and that between those blocks they would have the opportunity to be seated and take a drink of water (break duration was determined by participant comfort; average duration of each block was 6 min 22 $\mathrm{s}, \pm 48 \mathrm{~s} ; S D)$.

Blocks consisted of randomly selected trials from each auditory delay condition. The duration of each individual trial was $7 \mathrm{~s}$. This allowed each participant to take on average 11.3 footsteps ( \pm 1.1 footsteps; $S D$ ) in each delay condition. After each trial participants were instructed to respond by "yes" or "no" as to whether the walking that they heard over the headphones corresponded to the walking they had just performed. We analyzed the percentage of "yes" responses (agency judgment). We studied audio-motor agency based on experimental paradigms and agency questions that are comparable to those employed in previous work on visuo-motor agency (Fourneret \& Jeannerod, 1998; Franck et al., 2001; Kannape, Schwabe, Tadi, \& Blanke, in press; Shimada et al., 2010). 


\section{Materials}

The individual footsteps were recorded by microphones (Voice Technologies VT 500, Switzerland) that were attached to each shoe (tip; Figure 1). These recordings were presented binaurally, with a delay determined by the condition from which the trial was selected. Conditions were presented in randomized fashion and participants wore closed headphones with high ambient noise attenuation (BeyerDynamic DT 770M, Germany).

Auditory delays were implemented via custommade software on a portable computer (MacBook $2.16 \mathrm{GHz}$ Core 2 Duo, USA), carried by participants in a backpack. We tested 19 different temporal delays ranging from 16 to $1800 \mathrm{~ms}$ that were implemented "online" while participants were walking. Each condition was repeated 20 times and represented a different delay between the actual footstep and the presented auditory cue associated with the actual footstep. The tested delays are shown in Figure 2. The minimal delay the system was able to provide was $16 \mathrm{~ms}$. For each trial the gait period was determined from the

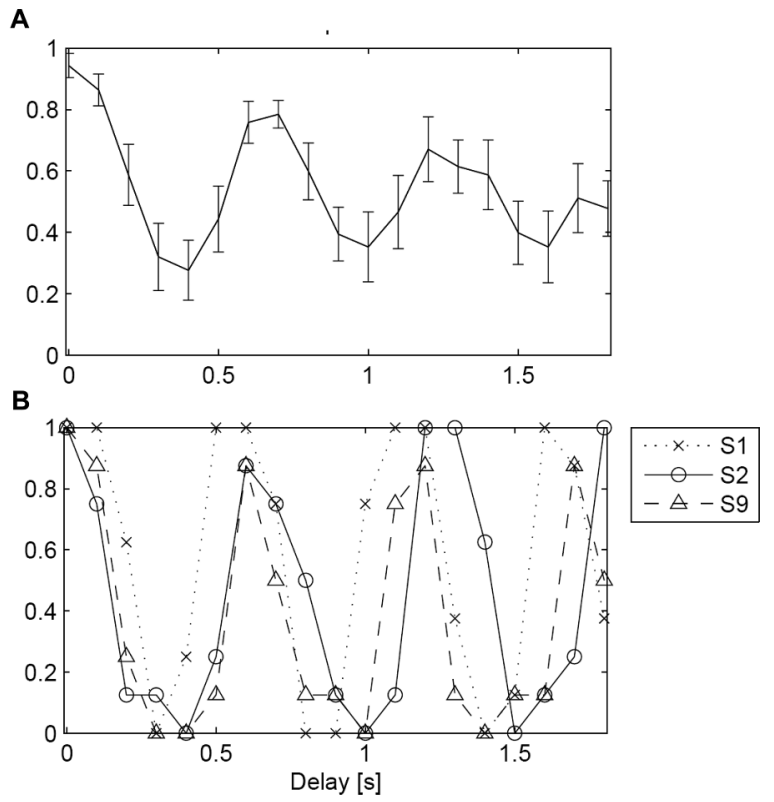

Figure 2. Uncorrected agency judgments. A. The plot shows agency judgments (mean $\pm 1 \mathrm{SD}$ ) across delay conditions. The data show that agency judgments depend on the delay (i.e. initial decrease in agency judgments until $\sim 450 \mathrm{~ms}$ ). The sinusoidal pattern suggests that the cyclic nature of walking also influences agency judgments. Note the dampening of the sinusoidal pattern with increasing delays. B. Individual data from three participants are shown (S1, S2, S9). These data show that agency judgments were as precise (and as elevated) as for the minimal delay of $16 \mathrm{~ms}$. Note that there is no apparent dampening of the agency curve. audio signal recorded by the microphones. The gait period was calculated as the position of the maximum of the autocorrelation of the signal's envelope outside of the range of $0-0.8 \mathrm{~s}$ (assuming that the gait period is $>0.8 \mathrm{~s}$ ). The custom software allowed for precision recording of the auditory profile of participants' footsteps over time. To exclude personal "shoe-specific" auditory signals, all participants wore the same pair of experimental shoes, adjusted for size via an ankle strap. A hand-held device (Wii Remote, Nintendo, Japan) was used to register, after each trial, the participant's agency judgment. For statistical analysis a within-subjects design was used.

\section{RESULTS}

\section{Agency judgments}

Data from the agency judgment task were collated across participants for each delay condition and are represented in Figure 2A, showing that agency judgments depend on delay. As predicted, the data reveal the highest percentage of confirmatory agency judgments $(\sim 90 \%)$ for delays of 16 and 100 ms which rapidly decrease to $34 \%$ and $28 \%$ for delays between 250 and $450 \mathrm{~ms}$. For $450-750 \mathrm{~ms}$ delays, the percentage of agency judgments increased continuously to $\sim 75 \%$. Over the next $750 \mathrm{~ms}$ this sinusoidal pattern was repeated (maximum of $\sim 65 \%$ at $1300 \mathrm{~ms}$ ). A 4 parameter damped sine wave model fitted to the data yields an $R^{2}$ value of .92 , thus explaining $92 \%$ of the variance (significant fit; $p<.05$ ). These data provide evidence of a predictable relationship between agency judgments and the extent of the auditory delay.

We next analyzed whether the damping with increasing delays reflects increased variability of the peaks of maximal and minimal agency judgments for larger delays. Inspection of individual data (Figure 2B) shows that agency judgments were as precise (and as elevated) as for the minimal delay of $16 \mathrm{~ms}$ in most participants, showing that variability does not account for dampening. Moreover, the sinusoidal pattern suggests that the cyclic nature of walking (Murray, 1967; Blanc Balmer, C., Landis, T., \& Vingerhoets,, 1999) interfered with agency judgments. Gait agency may depend not only on delay, but also on gait cycle and gait speed. All participants were instructed to walk at their habitual speed, but as stride length and walking speed depend on individualistic parameters (i.e., height and leg length; Macellari, Giacomozzi, \& Saggini, 1999), we next analyzed our participants' walking speed. 

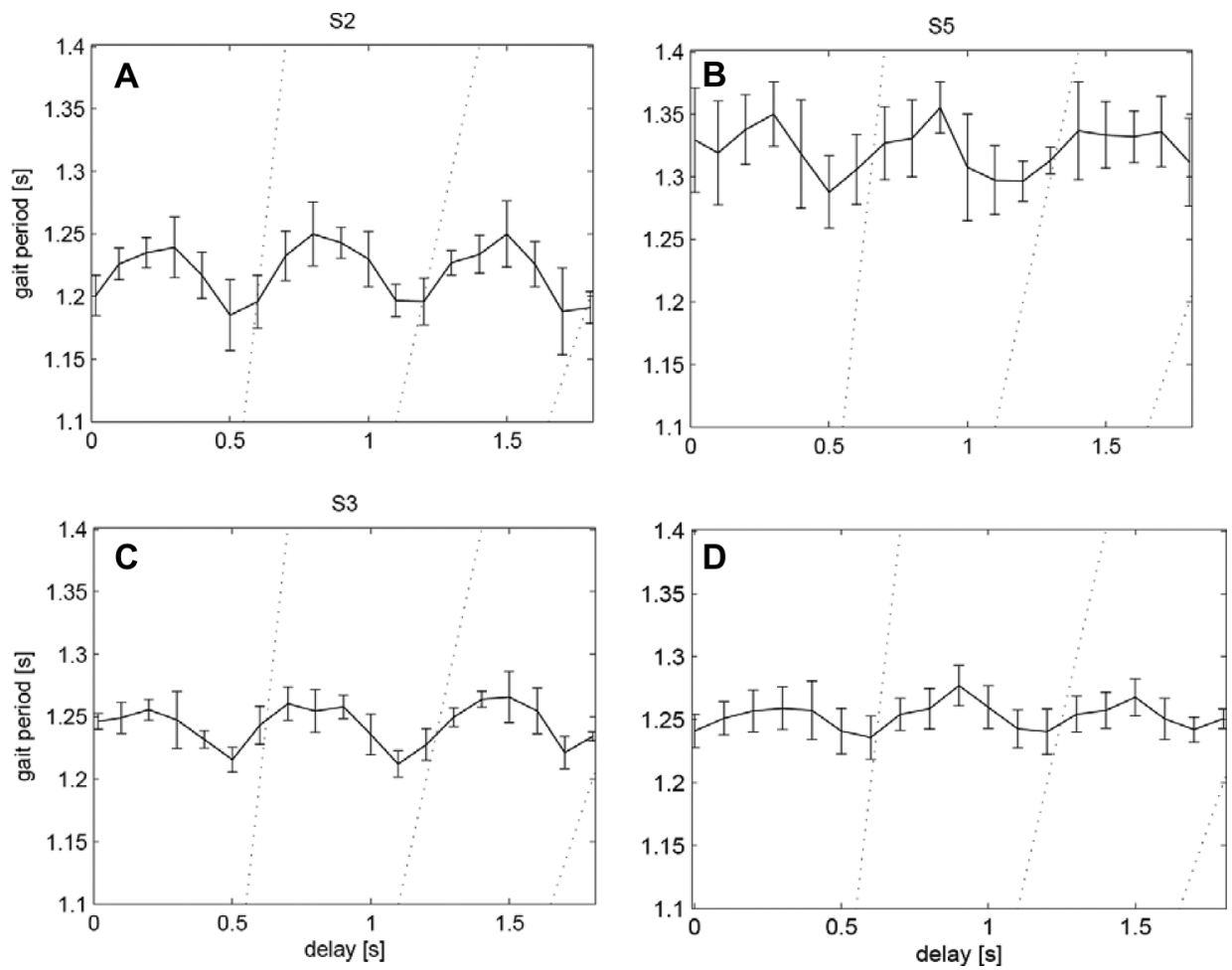

Figure 3. Gait period is shown as a function of delay condition for three individual subjects (A-C) and all participants (D). The average gait period showed small but systematic variation with the delay period in a sinusoidal pattern for the three depicted participants (S2, S3, S5). Because the participants with longer gait periods (higher curve on $y$-axis) also had slower varying gait period vs. delay curves (curve stretched along the $x$-axis), the average data across all participants (Figure 3D) were obtained by stretching the individual curves by the same factor in $x$ and $y$ direction until their mean was the same as the previously calculated mean gait period, and subsequently taking the mean over all stretched curves. Thus, before calculating the average curve, the curve of each participant was stretched along the dotted lines until their mean matched the average gait period across all participants. Note that the delay conditions with smallest gait period (or fastest walking speed) overlap with the delay conditions for which we found that agency judgments reached maximal values (compare with Figure 2A).

\section{Gait-period and walking speed depend on delay}

Analysing the gait period of each participant as a function of delay condition, we found that the average gait period was $1.25 \mathrm{~s}(0.13 \mathrm{~s}(S D)$; range: $1.01-1.79 \mathrm{~s})$ and that this varied systematically with the delay period in a sinusoidal pattern (Figure 3). On average participants' footsteps were separated by $613 \mathrm{~ms}$, compatible with physiological data in healthy subjects (Blanc, Balmer, Landis, \& Vingerhoets, 1999; Macellari et al., 1999). Figure 3 depicts that the gait period (walking speed) showed small variations as a function of the delay conditions. We found a first maximum of the gait period at $\sim 0.4 \mathrm{~s}$, followed by a minimum at $\sim 0.6 \mathrm{~s}$, a maximum at $\sim 0.9 \mathrm{~s}$, another minimum at $\sim 1.2 \mathrm{~s}$, and a final maximum at $\sim 1.5 \mathrm{~s}$. The delay conditions with smallest gait period (or fastest walking speed) overlapped with the delay conditions for which we found that agency judgments reached maximal values (compare with Figure 2A), comparable to those at the minimal delay $(16 \mathrm{~ms})$. These data show that participants' walking speed depended on the delay period, increasing after each stride and decreasing at each stride, although they were not aware of this (as revealed by questioning after the end of the experiment) and were instructed to walk at a normal habitual speed throughout the experiment.

\section{Gait-corrected agency judgments}

This makes it possible that the decrease in sine wave amplitude that we observed for agency judgments across delays is a consequence of the phase relationship between auditory delay and individual gait period. We converted the delay conditions (in s) to normalized delay conditions for each participant (the proportion of the delay with respect to the participant's gait period). The resulting data are represented in Figure 4. As can be seen in Figure 4, these gait-corrected 


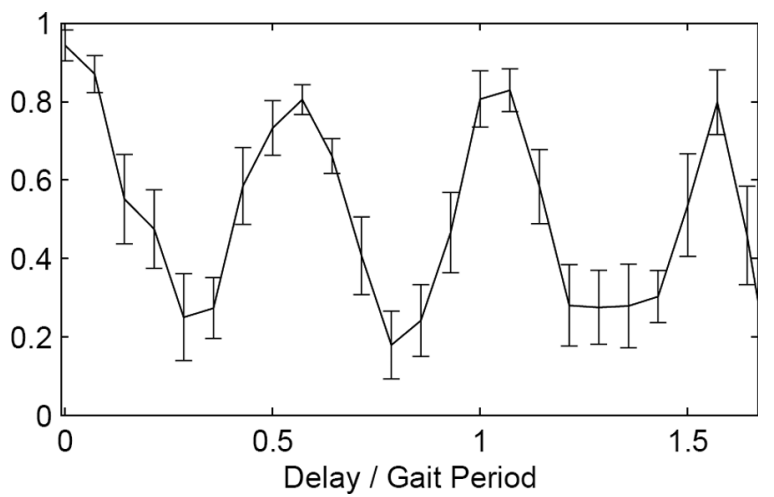

Figure 4. Gait-corrected agency judgments (mean $\pm 1 S D$ ) across normalized delay conditions. Delay conditions (in s) were converted to normalized delay conditions for each participant (the proportion of the delay with respect to the participant's gait period). Values on the $x$-axis represent the gait-normalized delay conditions and values on the $y$-axis show agency judgments. Concerning normalized delay conditions, 0 and 1 represent time points of objective simultaneity. That is, they represent the conditions in which auditory and sensorimotor events objectively were in phase (although for the 1.0 condition, the auditory signal participants heard while simultaneously taking a step with the left foot was actually generated from the previous left footstep). The 0.5 and 1.5 points also represent points of objective simultaneity, but this time with pedal (left/right) crossover. So, in these conditions, simultaneous to taking a left footstep, participants heard the auditory signal associated with a previous right footstep. Note that the absence of agency judgment decreases around the time points of objective simultaneity.

agency data show no decrease or damping in sine wave amplitude across delays, suggesting that the decrease observed for the gait-uncorrected agency data is due to an influence of the variable gait periods between averaged individuals. Gait-corrected agency data are best fit by a sine wave function, and this function explains a significant amount of the variance $\left(R^{2}\right.$ $=.92, p<.05)$. Based on this model, we note that at the points of objective simultaneity, participants reliably made confirmatory agency judgments (based on the model that was the case for approximately $75 \%$ of trials). Recalibration of the observed $75 \%$ threshold with actual gait periods suggests the range of delays across which perceived simultaneity reliably was reported to be $\sim 113 \mathrm{~ms}$.

\section{DISCUSSION}

\section{Auditory and visual effects on gait agency}

Confirmatory gait agency judgments (the percentage of "yes" responses) in the present experiment decreased rapidly for delays $>120 \mathrm{~ms}$ and reached a first minimum at $400-500 \mathrm{~ms}$. To the best of our knowledge, agency during gait movements has not been tested using either audio-motor or visuo-motor conflicts. Our data may be compared with those described by Sato and Yasuda (2005; Experiment 2) testing agency for hand movements using finger button presses and audio-motor conflicts. These authors also observed strongly diminished agency judgments for delays $>250 \mathrm{~ms}$ that further decreased until 600 $\mathrm{ms}$. Yet this comparison is hampered by the fact that the actions tested by Sato and Yasuda (2005) were single, goal-directed movements that were in addition associated with two different auditory action consequences (congruent and incongruent tones). This also applies to the comparison of our data with those reported by Asai \& Tanno (2008), who also used finger button presses and manipulated the timing of auditory action consequences, but reported a strong decrease in such agency judgments for delays $>\sim 100$ ms. Comparison with the interesting auditory agency data by Knoblich and Repp is also difficult, as experts and naïve participants were asked to detect the moment of transition between externally and self-controlled tapping sequences (Knoblich \& Repp, 2009; Repp \& Knoblich, 2007). Our data manipulating performance-related auditory cues are comparable with data manipulating performance-related visual cues. Thus, Franck et al. (2001) measured agency judgments of hand actions (joystick movements) and tested different delays between a movement and a visually presented movement. They observed mostly self agency judgments for delays $<100 \mathrm{~ms}$ and mostly non-self agency judgments for delays $>150 \mathrm{~ms}$. A comparable value of visual-motor delay has been reported by Farrer et al. (2008; Study 1; 120 ms) and Shimada et al. (2010; 230 ms) using similar hand movement tasks and feedback conditions. Collectively, these studies using visuo-motor and audiomotor conflict report similar values at which agency judgments decrease, $\sim 100-200 \mathrm{~ms}$. These findings are also similar to those employing spatial visuo-motor conflict to manipulate agency (i.e. Daprati et al., 1997; Fourneret \& Jeannerod, 1998; Franck et al., 2001; Knoblich \& Kircher, 2004; van den Bos \& Jeannerod, 2002).

This similarity when judging temporal delays between visuo-motor and audio-motor cues is surprising considering the excellent temporal resolution of the auditory system and the relatively poor temporal resolution of the visual system. Moreover, the delay values of $\sim 100-200 \mathrm{~ms}$ are far above the threshold for auditory temporal order judgments of $\sim 20 \mathrm{~ms}$ (Hirsh, 1959 and Hirsh \& Sherick, 1961; cited in Knoblich \& Repp, 2009). We therefore suggest that this similarity reflects common, supramodal, mechanisms in the 
conscious action monitoring of auditory and visual action consequences. This is compatible with a comparison of the predicted and actual consequences of actions and gait (Frith et al., 2000) or the presence of a dedicated "who-system" (Georgieff \& Jeannerod, 1998; Jeannerod, 2006) that is independent of the sensory modality tested. Given the relative rarity of studies testing the effects of performance-related auditory cues on agency, this has to be regarded with caution and may depend on the employed agency manipulation and task and may differ between explicit and implicit agency judgments (Repp \& Knoblich, 2007).

\section{Gait and bodily self-consciousness}

Although gait has been considered initially a largely automatic action regulated mainly by subcortical control mechanisms, recent work has highlighted the influence of attentional, executive and other cognitive mechanisms (Shaw, 2002; Yogev-Seligmann, Hausdorff, \& Giladi, 2008). Walking is a complex task involving the integration of locomotion, balance, and adaptation in an ever-changing environment (Armstrong, 1988; Blanc et al., 1999; Drew, Prentice, $\&$ Schepens, 2004). Moreover, the neuroscience of upright gait is hampered by two main caveats. Neuroimaging using functional magnetic resonance imaging (fMRI), magnetoencephalography (MEG), or electroencephalography (EEG) is currently not available or severely limited in walking humans (but see Fukuyama et al., 1997; Miyai et al., 2001) and humans are the only truly upright walking primates (i.e. Eccles, 1989). The neuroscience of walking is thus almost entirely based on findings in quadrupeds and behavioral work in patients with gait disorders, pointing to a distributed network including spinal cord, brainstem, basal ganglia, cerebellum, motor and posterior parietal cortex (motor cortex: Armstrong, 1988; Drew et al., 2004; spinal cord: Grillner \& Wallen, 1985; Nutt, Marsden, \& Thompson, 1993). The present data extend the data on cognitive gait mechanisms (Lundin-Olsson, Nyberg, \& Gustafson, 1997; Shaw, 2002; Yogev-Seligmann et al., 2008) by revealing systematic conscious contributions to gait control. This is compatible with findings suggesting that human gait is a complex higher form of movement characterized by many cognitive components and likely represented at the cortical level.

In the case of conscious control for arm or hand actions awareness is lacking for the movement of a certain body part (in general the arm or the hand) of the agent, but not for the position and locomotion of the agent's entire body. We have proposed that the "I" of conscious experience of the self - which has been linked to full-body representations (Blanke \& Metzinger, 2009) - is not altered in these agency manipulations of body parts. We propose that the present procedure allowed us to manipulate the "I" of conscious experience or global bodily self, extending previous work on full-body ownership to full-body agency (Ehrsson, 2007; Kannape et al., in press; Lenggenhager et al., 2007, 2009; Petkova and Ehrsson, 2008). However despite these different functional consequences of the movement of a person's body part or a person's entire body, the present experimental data suggest that humans rely on comparable mechanisms for monitoring the action of a single body part (i.e. arm) and their entire body.

\section{Periodic gait agency}

Due to the gait cycle and continuously alternating right and left footsteps, those delays approaching the time of the subsequent actual footstep were found to increase agency judgments. Our data using gait-correction show that participants under the present experimental conditions were not aware of such moments of objective simultaneity between auditory cues and subsequent actual footsteps. This was found for all moments of objective simultaneity (with and without pedal crossover). When, simultaneous to taking a left (right) footstep, participants heard the auditory signal associated with a previous right (left) footstep, they showed no agency judgment differences. This suggests that conscious gait monitoring, in addition to mechanisms leading to gradual changes in agency judgments, also depends on periodic changes in agency judgments that depend on the participant's gait period, independent of pedal crossover. Periodic agency mechanisms have also been described in trained and naïve subjects during rhythmic tapping movements (Knoblich \& Repp, 2009; Repp \& Knoblich, 2007). We speculate that the reported independence of pedal crossover is only present in "experts" in rhythmic stepping movements and that there will be no comparable or less strong effects of manual crossover in tapping studies.

Our data show that it is important to include the actual movement in analysis, revealing that participants modified their walking speed depending on the tested delay condition. Moreover, we found a highly systematic influence of delay condition on walking speed, leading to an increase for auditory delays that were shorter than the first, second, and third subsequent footstep; followed by a decrease for auditory delays that were longer than the first, second, and third subsequent footstep. The automatic modulation 
of gait period by auditory delay is reminiscent of some cognitive effects on gait (Shaw, 2002; YogevSeligmann et al., 2008) as dual task performance (i.e., counting or speaking while waking) may lead to changes in walking speed that may in some subjects (especially the elderly) even lead to gait arrest and falling (Lundin-Olsson et al., 1997; Yogev-Seligmann et al., 2008). The automatic modulation of gait period is also reminiscent of spatial corrections of hand movement trajectories during hand agency judgments (Fourneret \& Jeannerod, 1998; Nielsen, 1963) for which subjects are unaware.

\section{CONCLUSION}

The purpose of the present study was to investigate agency for the entire body by testing auditory action effects related to gait. For this we designed a portable stimulation and recording system in combination with performance-related auditory cues, allowing us to induce online sensorimotor conflicts and changes in agency judgments in moving agents. We show that the control of full-body locomotion and the building of a conscious experience of it are at least partially distinct brain processes. A comparison with the previous literature revealed that these delay-related agency mechanisms were similar whether auditory or visual consequences of actions were tested, compatible with supramodal, modality-independent control mechanisms. We argue that the further study of agency and ownership for a person's full body may help to refine our scientific criteria of selfhood (Blanke \& Metzinger, 2009) and are of relevance for neurological conditions (Arzy, Seeck, Ortigue, Spinelli, \& Blanke, 2006; Blanke et al., 2003) and psychiatric conditions (Daprati et al., 1997; Franck et al., 2001) characterized by a disturbance of selfhood.

\section{REFERENCES}

Armstrong, D. M. (1988). The supraspinal control of mammalian locomotion. Journal of Physiology, 405, 1-37.

Arzy, S., Seeck, M., Ortigue, S., Spinelli, L., \& Blanke, O. (2006). Induction of an illusory shadow person. Nature, 443(7109), 287.

Asai, T., \& Tanno, Y. (2008). Highly schizotypal students have a weaker sense of self-agency. Psychiatry and Clinical Neurosciences, 62(1), 115-119.

Aspell, J. E., Lenggenhager, B., \& Blanke, O. (2009). Keeping in touch with one's self: Multisensory mechanisms of self-consciousness. PLoS One, 4(8), e6488.

Blanc, Y., Balmer, C., Landis, T., \& Vingerhoets, F. (1999). Temporal parameters and patterns of the foot roll over during walking: Normative data for healthy adults. Gait Posture, 10(2), 97-108.

Blanke, O., \& Metzinger, T. (2009). Full-body illusions and minimal phenomenal selfhood. Trends in Cognitive Sciences, 13(1), 7-13.

Blanke, O., Ortigue, S., Coeytaux, A., Martory, M. D., \& Landis, T. (2003). Hearing of a presence. Neurocase, 9(4), 329-339.

Daprati, E., Franck, N., Georgieff, N., Proust, J., Pacherie, E., Dalery, J., et al. (1997). Looking for the agent: An investigation into consciousness of action and self-consciousness in schizophrenic patients. Cognition, 65(1), 71-86.

Drew, T., Prentice, S., \& Schepens, B. (2004). Cortical and brainstem control of locomotion. Progress in Brain Research, 143, 251-261.

Eccles, J. C. (1989). Evolution of the hominid brain: Bipedality; agility. In J. C. Eccles (Ed.), Evolution of the brain: Creation of the self (Vol. 1, pp. 39-69). London, UK: Routledge.

Ehrsson, H. H. (2007). The experimental induction of out-of-body experiences. Science, 317(5841), 1048.

Farrer, C., Franck, N., Georgieff, N., Frith, C. D., Decety, J., \& Jeannerod, M. (2003). Modulating the experience of agency: A positron emission tomography study. Neuroimage, 18(2), 324-333.

Farrer, C., Frey, S. H., Van Horn, J. D., Tunik, E., Turk, D., Inati, S., et al. (2008). The angular gyrus computes action awareness representations. Cerebral Cortex, $18(2), 254-261$.

Fourneret, P., \& Jeannerod, M. (1998). Limited conscious monitoring of motor performance in normal subjects. Neuropsychologia, 36(11), 1133-1140.

Franck, N., Farrer, C., Georgieff, N., Marie-Cardine, M., Dalery, J., d'Amato, T., et al. (2001). Defective recognition of one's own actions in patients with schizophrenia. American Journal of Psychiatry, 158(3), 454-459.

Frith, C. D., Blakemore, S. J., \& Wolpert, D. M. (2000) Abnormalities in the awareness and control of action. Philosophical Transactions of the Royal Society of London, Series B: Biological Sciences, 355(1404), 1771-1788.

Fukuyama, H., Ouchi, Y., Matsuzaki, S., Nagahama, Y., Yamauchi, H., Ogawa, M., et al. (1997). Brain functional activity during gait in normal subjects: A SPECT study. Neuroscience Letters, 228(3), 183-186.

Georgieff, N., \& Jeannerod, M. (1998). Beyond consciousness of external reality: A "who" system for consciousness of action and self-consciousness. Consciousness and Cognition, 7(3), 465-477.

Grillner, S., \& Wallen, P. (1985). Central pattern generators for locomotion, with special reference to vertebrates. Annual Review of Neuroscience, 8, 233-261.

Hirsh, I. J. (1959). Auditory perception of temporal order. Journal of the Acoustical Society of America, 31, 759-767.

Hirsh, I. J., \& Sherrick, C. E. (1961). Perceived order in different sense modalities. Journal of Experimental Psychology, 62, 423-432.

Jeannerod, M. (2006). The origin of voluntary action: History of a physiological concept. Comptes Rendus Biologies, 329(5-6), 354-362.

Jeannerod, M. (2007). Being oneself. Journal of Physiology (Paris), 101(4-6), 161-168.

Kannape, O. A., Schwabe, L., Tadi, T., \& Blanke, O. (in press). The limits of agency in walking humans. Neuropsychologia. 
Knoblich, G., \& Kircher, T. T. (2004). Deceiving oneself about being in control: Conscious detection of changes in visuomotor coupling. Journal of Experimental Psychology: Human Perception and Performance, 30(4), 657-666.

Knoblich, G., \& Repp, B. H. (2009). Inferring agency from sound. Cognition, 111(2), 248-262.

Lenggenhager, B., Mouthon, M., \& Blanke, O. (2009). Spatial aspects of bodily self-consciousness. Consciousness and Cognition, 18(1), 110-117.

Lenggenhager, B., Tadi, T., Metzinger, T., \& Blanke, O. (2007). Video ergo sum: Manipulating bodily self-consciousness. Science, 317(5841), 1096-1099.

Lundin-Olsson, L., Nyberg, L., \& Gustafson, Y. (1997). "Stops walking when talking" as a predictor of falls in elderly people. Lancet, 349(9052), 617.

Macellari, V., Giacomozzi, C., \& Saggini, R. (1999). Spatial-temporal parameters of gait: Reference data and a statistical method for normality assessment. Gait Posture, 10(2), 171-181.

Miyai, I., Tanabe, H. C., Sase, I., Eda, H., Oda, I., Konishi, I., et al. (2001). Cortical mapping of gait in humans: A nearinfrared spectroscopic topography study. NeuroImage, 14(5), 1186-1192.

Murray, M. P. (1967). Gait as a total pattern of movement. American Journal of Physical Medicine, 46, 290-333.

Nielsen, T. (1963). Volition: A new experimental approach. Scandinavian Journal of Psychology, 4(4), 225-230.
Nutt, J. G., Marsden, C. D., \& Thompson, P. D. (1993). Human walking and higher-level gait disorders, particularly in the elderly. Neurology, 43(2), 268-279.

Petkova, V. I., \& Ehrsson, H. H. (2008). If I were you: Perceptual illusion of body swapping. PLoS One, 3(12), e3832.

Repp, B. H., \& Knoblich, G. (2007). Toward a psychophysics of agency: Detecting gain and loss of control over auditory action effects. Journal of Experimental Psychology: Human Perception and Performance, 33(2), 469-482.

Sato, A., \& Yasuda, A. (2005). Illusion of sense of self-agency: Discrepancy between the predicted and actual sensory consequences of actions modulates the sense of self-agency, but not the sense of self-ownership. Cognition, 94(3), 241-255.

Shaw, F. E. (2002). Falls in cognitive impairment and dementia. Clinics in Geriatric Medicine, 18(2), 159-173.

Shimada, S., Qi, Y., \& Hiraki, K. (2010). Detection of visual feedback delay in active and passive self-body movements. Experimental Brain Research, 201, 359-364.

Tsakiris, M., Haggard, P., Franck, N., Mainy, N., \& Sirigu, A. (2005). A specific role for efferent information in self-recognition. Cognition, 96(3), 215-231.

van den Bos, E., \& Jeannerod, M. (2002). Sense of body and sense of action both contribute to self-recognition. Cognition, 85(2), 177-187.

Yogev-Seligmann, G., Hausdorff, J. M., \& Giladi, N. (2008). The role of executive function and attention in gait. Movement Disorders, 23(3), 329-342; quiz 472. 